\title{
Longevidade e parâmetros reprodutivos de Myzus persicae (Sulzer, 1776) (Hemiptera: Aphididae) sobre berinjela em diferentes temperaturas
}

\author{
Longevity and reproductive parameters of Myzus persicae (Sulzer, 1776) \\ (Hemiptera: Aphididae) on eggplant at different temperatures
}

\author{
Marcos Doniseti Michelotto ${ }^{1}$ Norton Rodrigues Chagas Filho ${ }^{2}$ \\ Ricardo Adaime da Silva ${ }^{3}$ Antonio Carlos Busoli ${ }^{4}$
}

\section{RESUMO}

O afídeo Myzus persicae (Sulzer, 1776) (Hemiptera: Aphididae) é uma das principais pragas de diversas culturas em condições de campo ou em cultivo protegido. Este trabalho teve por objetivo avaliar o efeito de diferentes temperaturas na longevidade e nos aspectos reprodutivos de M. persicae sobre berinjela (Solanum melongena L.). $O$ experimento foi conduzido sob condições controladas de temperatura $\left(15,20,25\right.$ e $\left.30^{\circ} \mathrm{C}\right)$, umidade relativa do ar $(70 \pm$ $10 \%)$ e fotofase (12 horas). As unidades experimentais consistiram de placas de Petri contendo ágar-água solidificado (1\%). Nestas placas, os afídeos foram mantidos individualmente sobre os discos foliares de berinjela ( $3 \mathrm{~cm}$ de diâmetro) em uma das temperaturas (tratamentos), com 25 repetições. Foram determinadas as curvas mais ajustadas aos parâmetros biológicos de $\boldsymbol{M}$. persicae, suas equações de regressão e os respectivos coeficientes de determinação $\left(R^{2}\right)$. A temperatura influenciou todos os parâmetros avaliados. As durações dos períodos pré-reprodutivo e reprodutivo de $\boldsymbol{M}$. persicae variaram de 0,46 dia $\left(25^{\circ} \mathrm{C}\right)$ a 1,12 dia $\left(15^{\circ} \mathrm{C}\right)$ e de 3,89 dias $\left(25^{\circ} \mathrm{C}\right)$ a 19,11 dias $\left(15^{\circ} \mathrm{C}\right)$, respectivamente. A fecundidade total e diária foi de 17,63 ninfas/fêmea e 4,38 ninfas/fêmea/dia a $25^{\circ} \mathrm{C}$; 43,63 ninfas/fêmea e 4,34 ninfas/fêmea/dia a $20^{\circ} \mathrm{C} ; 60,65$ ninfas/fêmea e 3,15 ninfas/fêmea/dia a $15^{\circ} \mathrm{C}$. A $30^{\circ} \mathrm{C}$, não houve reprodução. A duração do período pós-reprodutivo variou de 0,89 dia $\left(25^{\circ} \mathrm{C}\right)$ a 3,72 dias $\left(15^{\circ} \mathrm{C}\right)$. A longevidade do afídeo diminuiu com o aumento da temperatura, de 23,95 dias $\left(15^{\circ} \mathrm{C}\right)$ para 5,06 dias $\left(25^{\circ} \mathrm{C}\right)$. Temperaturas entre 15 e $20^{\circ} \mathrm{C}$ são mais favoráveis a M. persicae.

Palavras-chave: Solanum melongena, afídeo, biologia, reprodução.

\begin{abstract}
The aphid Myzus persicae (Sulzer, 1776) (Hemiptera: Aphididae) is one of the main pests in a number of crops both under field and protected conditions. The objective of this work was to study the effect of temperature on longevity and reproductive parameters of $\boldsymbol{M}$. persicae on eggplant (Solanum melongena L.). The experiment was carried out under controlled conditions of temperature $\left(15,20,25\right.$ and $\left.30^{\circ} \mathrm{C}\right)$, relative humidity $(70 \pm 10 \%)$ and photophase (12 hours). The experimental units consisted of Petri dishes containing agar-water (1\%) solidified. Aphids were kept individually on leaf discs of eggplant (3 $\mathrm{cm}$ diameter) at each temperature, with 25 replicates. Curves more adjusted to the biological parameters of $\boldsymbol{M}$. persicae, their regression equations and respective determination coefficients $\left(R^{2}\right)$ were determined. All biological parameters were affected by temperature. The prereproductive and reproductive periods of $\mathbf{M}$. persicae varied from 0.46 day $\left(25^{\circ} \mathrm{C}\right)$ to 1.12 day $\left(15^{\circ} \mathrm{C}\right)$ and from 3.89 days $\left(25^{\circ} \mathrm{C}\right)$ to 19.11 days $\left(15^{\circ} \mathrm{C}\right)$, respectively. The total and daily fecundity was 17.63 and 4.38; 43.63 and 4.34; and 60.65 nymphs/female and 3.15 nymphs/female/day, respectively, at 25,20 and $15^{\circ} \mathrm{C}$. At $30^{\circ} \mathrm{C}$ there was no reproduction. The postreproductive period varied from 0.89 day $\left(25^{\circ} \mathrm{C}\right)$ to 3.72 days $\left(15^{\circ} \mathrm{C}\right)$. The longevity of the aphid decreased with the increase in temperature, from 23.95 days $\left(15^{\circ} \mathrm{C}\right)$ to 5.06 days $\left(25^{\circ} \mathrm{C}\right)$. Thus, temperatures between 15 and $20^{\circ} \mathrm{C}$ are more favorable to $M$. persicae.
\end{abstract}

Key words: Solanum melongena, aphid, biology, reproduction.

${ }^{1}$ Doutorando em Entomologia Agrícola, Faculdade de Ciências Agrárias e Veterinárias (FCAV), Universidade Estadual Paulista (UNESP), Via de Acesso Prof. Paulo Donato Castellane, s/n, 14884-900, Jaboticabal, São Paulo, Brasil. E-mail: michelot@fcav.unesp.br. Autor para corresondência.

${ }^{2}$ Mestrando em Entomologia Agrícola, FCAV, UNESP, Jaboticabal, São Paulo, Brasil.

${ }^{3}$ Pesquisador da Embrapa Amapá, Rodovia JK, km 5, 68903-000, Macapá, Amapá, Brasil.

${ }^{4}$ Departamento de Fitossanidade, FCAV, UNESP, Jaboticabal, São Paulo, Brasil. E-mail: acbusoli@fcav.unesp.br. 


\section{INTRODUÇÃO}

A berinjela (Solanum melongena L.) é uma solanácea originária das regiões tropicais do continente asiático, sendo cultivada há muitos séculos por chineses e árabes (FILGUEIRA, 2002). Embora a área plantada no Brasil seja pouco superior a 1500ha, tem ocorrido um aumento no consumo da olerícola, motivado pela procura por parte dos consumidores de produtos mais saudáveis e com propriedades medicinais. Neste aspecto, a berinjela destaca-se pela sua propriedade redutora do nível de colesterol (PINTO et al., 2000). Dentre os fatores que podem reduzir a produtividade da berinjela, encontram-se os insetos, que danificam as diferentes partes da planta, sendo geralmente controlados com inseticidas (GALLO et al., 2002).

O afídeo Myzus persicae (Sulzer, 1776) (Hemiptera: Aphididae) possui ampla distribuição mundial e encontra-se associado a culturas de grande importância econômica. É uma espécie polífaga, capaz de transmitir mais de 100 vírus fitopatogênicos (BLACKMAN \& EASTOP, 1984). Na cultura da berinjela, é transmissor do Potato Vírus Y (PVY), que causa severos danos às plantas, reduzindo drasticamente a produtividade na maioria das cultivares (PINTO et al., 2000). Plantas infectadas por PVY apresentam folhas com sintomas de mosaico, amareladas e de tamanho reduzido, além de frutos pouco desenvolvidos.

Diversos fatores podem afetar a reprodução dos afídeos, tais como a qualidade da planta hospedeira, a ocorrência natural de resistência (diferença entre as cultivares) e a temperatura (KOCOUREK et al., 1994). DIXON (1987) relata que uma mudança na temperatura resulta em alteração na taxa reprodutiva e na longevidade dos afídeos.

O objetivo deste trabalho foi avaliar o efeito de diferentes temperaturas nos parâmetros reprodutivos e na longevidade de $\boldsymbol{M}$. persicae sobre folhas de berinjela.

\section{MATERIAL E MÉTODOS}

O experimento foi conduzido no Laboratório de Controle Biológico de Insetos do Departamento de Fitossanidade, setor de Entomologia da FCAV-UNESP, em Jaboticabal, Estado de São Paulo. Foram utilizadas quatro câmaras climatizadas com temperaturas de 15, 20, 25 e $30 \pm 1^{\circ} \mathrm{C}$, umidade relativa de $70 \pm 10 \%$ e fotofase de 12 horas. Foi utilizada a mesma metodologia de MICHELOTTO \& BUSOLI(2003).
Criação de manutenção dos afídeos

As sementes de berinjela foram semeadas em vasos de plástico com capacidade para 5 litros, contendo terra, areia e esterco na proporção 2:1:1, e mantidas em gaiolas de $1,5 \mathrm{~m} \times 1,5 \mathrm{~m}$, revestidas com uma tela anti-afídeo. Os afídeos utilizados no experimento foram coletados em colônias presentes em plantas de berinjela no campo, sendo posteriormente transferidos para as plantas (50 dias de idade) com auxílio de um pincel, para que se alimentassem e se reproduzissem.

Obtenção de adultos na fase reprodutiva

Foram coletadas folhas de berinjela contendo afídeos provenientes das colônias de criação e manutenção, e levados até o laboratório. Para cada temperatura foram preparadas três placas de Petri $(6 \mathrm{~cm}$ de diâmetro) contendo 15mL de ágar-água (1\%) solidificado e um disco foliar de berinjela ( $3 \mathrm{~cm}$ de diâmetro) disposto no centro da placa. Antes de sua utilização, as folhas foram lavadas em água corrente e deixadas imersas em solução de hipoclorito de sódio a $1 \%$ por um minuto e, posteriormente, lavadas em água corrente. Após a assepsia, as folhas foram secas em papel absorvente e, utilizando-se um vazador, foram obtidos os discos foliares. Em cada disco foliar de berinjela, foram colocados, com auxílio de um pincel, cinco adultos ápteros de $\boldsymbol{M}$. persicae. A tampa dessas placas continha uma abertura de $3 \mathrm{~cm}$ de diâmetro, coberta com uma tela anti-afídeo para permitir a aeração e evitar a fuga dos insetos. As placas foram identificadas e mantidas nas câmaras climatizadas, nas diferentes temperaturas, sendo vistoriadas três vezes ao dia para a obtenção das ninfas, as quais foram observadas até se tornarem adultas.

Delineamento experimental, condução e avaliação do experimento

O experimento foi realizado em delineamento inteiramente casualizado, cada temperatura $\left(15,20,25\right.$ e $\left.30^{\circ} \mathrm{C} \pm 1^{\circ} \mathrm{C}\right)$ correspondendo a um tratamento. Foram utilizadas 25 placas de Petri (repetições) contendo os discos foliares. Para cada placa, foi transferida uma ninfa, com auxílio de um pincel, sendo tampada e identificada. As avaliações foram realizadas a cada doze horas, sendo efetuada a transferência do afídeo para uma nova placa quando observados os primeiros sinais de deterioração das folhas. Foram avaliadas a duração dos períodos préreprodutivo, reprodutivo e pós-reprodutivo, a longevidade e a fecundidade total e diária das fêmeas. Os dados relativos a cada parâmetro avaliado foram submetidos à análise de variância, 
sendo as médias dos tratamentos comparadas pelo teste de Tukey a 5\% de probabilidade de erro. Os dados referentes aos períodos préreprodutivo e pós-reprodutivo, em dias, foram transformados em $(x+0,5)^{1 / 2}$, em função da ocorrência de valores relativamente baixos e alguns nulos. Foram determinadas as curvas mais ajustadas aos parâmetros reprodutivos dos afídeos, suas equações de regressão e os respectivos coeficientes de determinação $\left(R^{2}\right)$.

\section{RESULTADOS E DISCUSSÃO}

A temperatura influenciou os parâmetros reprodutivos e a longevidade de $\boldsymbol{M}$. persicae (Tabelas 1 e 2 e Figura 1), corroborando as afirmações de VAN EMDEN et al. (1969), de que a temperatura é fator extremamente importante no desenvolvimento dos afídeos.

Na temperatura de $30^{\circ} \mathrm{C}$, apenas duas ninfas atingiram a fase adulta, as quais duraram em média 2,45 dias e estas fêmeas não produziram ninfas. Assim, esta temperatura não é mencionada nos próximos parâmetros avaliados. BARLOW (1962) observou que indivíduos das espécies $\boldsymbol{M}$. persicae e Macrosiphum euphorbiae (Thomas, 1878), em batata (Solanum tuberosum L.), mantidos a $30^{\circ} \mathrm{C}$, morreram antes de se tornarem adultos. Em couve (Brassica oleracea L.), CIVIDANES \& SOUZA (2003) também observaram $100 \%$ de mortalidade das ninfas de $\boldsymbol{M}$. persicae, sugerindo que esta espécie não está adaptada a elevadas temperaturas $\left(30^{\circ} \mathrm{C}\right)$ e que o limite térmico superior de desenvolvimento (Ts) dessa espécie deve situar-se abaixo dessa temperatura.

\section{Período pré-reprodutivo.}

A duração do período pré-reprodutivo de fêmeas ápteras de $\boldsymbol{M}$. persicae decresceu com o aumento da temperatura (Tabela 1). A duração média desse período, em função da temperatura, demonstrou seguir o modelo linear (Tabela 1 e Figura 1), indicando que à medida que se aumenta a temperatura de 15 para $25^{\circ} \mathrm{C}$, ocorre uma redução linear $\left(\mathrm{R}^{2}=0,9758\right)$ na duração do período pré-reprodutivo. XIA \& TINGEY (1986), ao estudarem $\boldsymbol{M}$. persicae sobre dois híbridos de batata (Solanum tuberosum x Solanum bertaultii), “Superior” e D 888-4, observaram pequena duração do período pré-reprodutivo, com 0,88 e 1,13 dia nas temperaturas de $22: 15^{\circ} \mathrm{C}$ (dia/noite), respectivamente, e 0,79 e 1,08 dia nas temperaturas de $32: 18^{\circ} \mathrm{C}$ (dia/noite) para os híbridos "Superior" e D 888-4, respectivamente. NARVÁEZ \& NOTZ (1993) verificaram que a 26,71
Tabela 1 - Duração média (dias) dos períodos pré-reprodutivo, reprodutivo e pós-reprodutivo, e longevidade de $\boldsymbol{M}$. persicae sobre berinjela em diferentes temperaturas. Jaboticabal, SP. 2003.

\begin{tabular}{|c|c|c|c|c|}
\hline \multirow[b]{2}{*}{ Temperatura } & \multicolumn{3}{|c|}{ Períodos ${ }^{1,2}$} & \multirow[b]{2}{*}{ Longevidade $^{1,2}$} \\
\hline & $\begin{array}{c}\text { Pré- } \\
\text { reprodutivo }\end{array}$ & Reprodutivo & $\begin{array}{c}\text { Pós- } \\
\text { reprodutivo }\end{array}$ & \\
\hline $15^{\circ} \mathrm{C}$ & $\begin{array}{c}1,12 \pm 0,11 \text { a } \\
(n=23)\end{array}$ & $\begin{array}{c}19,11 \pm 1,37 a \\
(n=23)\end{array}$ & $\begin{array}{c}3,72 \pm 0,79 \text { a } \\
(n=23)\end{array}$ & $\begin{array}{c}23,95 \pm 1,77 a \\
(n=23)\end{array}$ \\
\hline $20^{\circ} \mathrm{C}$ & $\begin{array}{c}0,88 \pm 0,10 a \\
(n=24)\end{array}$ & $\begin{array}{c}10,23 \pm 1,10 b \\
(n=24)\end{array}$ & $\begin{array}{c}1,10 \pm 0,24 b \\
(n=24)\end{array}$ & $\begin{array}{c}12,21 \pm 1,19 b \\
(n=24)\end{array}$ \\
\hline $25^{\circ} \mathrm{C}$ & $\begin{array}{c}0,46 \pm 0,08 b \\
(n=16)\end{array}$ & $\begin{array}{c}3,89 \pm 0,62 \quad c \\
(n=16)\end{array}$ & $\begin{array}{c}0,89 \pm 0,16 \quad b \\
(n=16)\end{array}$ & $\begin{array}{c}5,06 \pm 0,62 \quad c \\
(n=17)\end{array}$ \\
\hline Média & $\begin{array}{c}0,86 \pm 0,07 \\
(n=63)\end{array}$ & $\begin{array}{c}11,99 \pm 0,62 \\
(n=63)\end{array}$ & $\begin{array}{c}2,02 \pm 0,34 \\
(n=63)\end{array}$ & $\begin{array}{c}14,53 \pm 1,24 \\
(n=64)\end{array}$ \\
\hline Teste F & $11,30 *$ & $40,39 *$ & $12,09 *$ & $45,39 *$ \\
\hline CV (\%) & 16,40 & 44,87 & 41,25 & 43,90 \\
\hline
\end{tabular}

${ }^{1}$ Média \pm erro padrão.

${ }^{2}$ Médias não seguidas pela mesma letra na coluna diferem entre si pelo teste de Tukey a 5\% de probabilidade de erro.

$\mathrm{n}=$ número de repetições.

* significativo a 5\% de probabilidade de erro.

Tabela 2 - Fecundidade diária e total de $\boldsymbol{M}$. persicae sobre berinjela em diferentes temperaturas. Jaboticabal, SP. 2003.

\begin{tabular}{lcc}
\hline Temperatura & $\begin{array}{c}\text { Fecundidade diária } \\
\text { (ninfas/fêmea/dia) }\end{array}$ & $\begin{array}{c}\text { Fecundidade total } \\
\text { (ninfas/fêmea) }\end{array}$ \\
\hline $15^{\circ} \mathrm{C}$ & $\begin{array}{c}3,15 \pm 0,15 \mathrm{~b} \\
(\mathrm{n}=23)\end{array}$ & $\begin{array}{c}60,65 \pm 4,57 \mathrm{a} \\
(\mathrm{n}=23)\end{array}$ \\
$20^{\circ} \mathrm{C}$ & $4,34 \pm 0,23$ a & $43,63 \pm 4,64 \mathrm{~b}$ \\
& $(\mathrm{n}=24)$ & $(\mathrm{n}=24)$ \\
$25^{\circ} \mathrm{C}$ & $4,38 \pm 0,33$ a & $17,63 \pm 3,12 \mathrm{c}$ \\
& $(\mathrm{n}=16)$ & $(\mathrm{n}=16)$ \\
\hline Média & $3,87 \pm 0,14$ & $43,50 \pm 3,31$ \\
& $(\mathrm{n}=63)$ & $(\mathrm{n}=63)$ \\
\hline Teste F & $9,29 *$ & $23,76 *$ \\
\hline $\mathrm{CV}$ (\%) & 27,24 & 28,48 \\
\hline
\end{tabular}

${ }^{1}$ Média \pm erro padrão.

${ }^{2}$ Médias não seguidas pela mesma letra na coluna diferem significativamente entre si pelo teste de Tukey a 5\% de probabilidade de erro.

n= número de repetições.

* significativo a $5 \%$ de probabilidade de erro. 


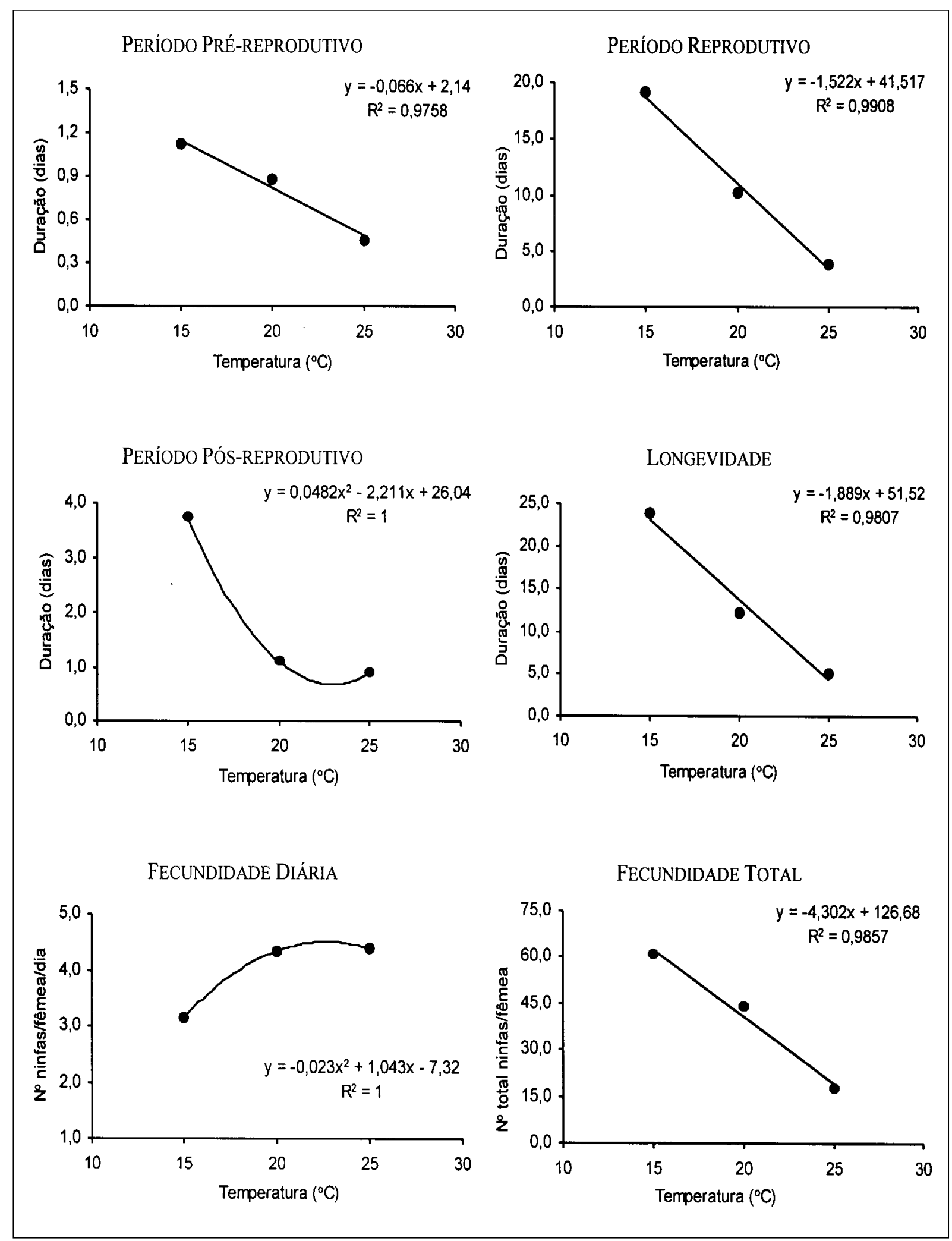

Figura 1 - Curvas ajustadas para a regressão entre a temperatura e a duração dos períodos pré-reprodutivo, reprodutivo e pósreprodutivo; a longevidade; a fecundidade diária e total de $\boldsymbol{M}$. persicae sobre berinjela. Jaboticabal, SP, 2003.

Ciência Rural, v. 35, n. 4, jul-ago, 2005. 
$\pm 3,02^{\circ} \mathrm{C}$ o período pré-reprodutivo de $\boldsymbol{M}$. persicae teve duração de 1,4 dia, em folhas de batata e de gergelim (Sesamum indicum L.).

Período reprodutivo.

O período reprodutivo de $\boldsymbol{M}$. persicae sofreu influência das temperaturas estudadas, seguindo um modelo linear (Figura 1). Dessa forma, a duração do período decresceu com aumento da temperatura, de 19,11 dias $\left(15^{\circ} \mathrm{C}\right)$ para 3,89 dias $\left(25^{\circ} \mathrm{C}\right)$ (Tabela 1$)$. NARVÁEZ \& NOTZ (1993) observaram variação para o período reprodutivo de $\boldsymbol{M}$. persicae, tendo como substrato folhas de batata e gergelim, com durações médias de 7,4 e 16,1 dias, respectivamente, sob temperatura de $26,71 \pm 3,02^{\circ} \mathrm{C}$. SOGLIA et al. (2003) observaram resultados semelhantes ao submeter Aphis gossypii Glover, 1877 a diferentes temperaturas e cultivares de crisântemo (Dendranthema grandiflora Tzvelev), com duração desse período decrescendo de 17,3 dias $\left(15^{\circ} \mathrm{C}\right)$ para 3,3 dias $\left(30^{\circ} \mathrm{C}\right)$.

Fecundidade.

A fecundidade diária de fêmeas ápteras de M. persicae, em função da temperatura, seguiu modelo quadrático (Figura 1). As fêmeas mantidas a 20 e $25^{\circ} \mathrm{C}$ produziram 4,34 e 4,38 ninfas/fêmea/dia, respectivamente, mais que aquelas mantidas na temperatura de $15^{\circ} \mathrm{C}$, com 3,15 ninfas/fêmea/dia (Tabela 2). Esses resultados são superiores aos observados por BASTOS et al. (1996), segundo os quais a fecundidade diária de $\boldsymbol{M}$. persicae na temperatura de $25^{\circ} \mathrm{C}$ foi de 1,63 ninfas/fêmea/dia, sobre couve, e aos observados por CIVIDANES \& SOUZA (2003), nas temperaturas de 15,20 e $25^{\circ} \mathrm{C}$ (2,0; 1,7 e 1,3 ninfas/fêmea/dia, respectivamente), para a mesma espécie e também sobre couve.

Com relação ao efeito das temperaturas estudadas sobre a fecundidade total, observou-se que, com o aumento da temperatura, ocorreu diminuição da fecundidade das fêmeas, ajustando-se a um modelo linear (Figura 1). Segundo este modelo, a fecundidade máxima ocorreu a $15^{\circ} \mathrm{C}$, com 60,65 ninfas/fêmea, e a menor na temperatura de $25^{\circ} \mathrm{C}$, com 17,63 ninfas/ fêmea. BASTOS et al. (1996) e CULLINEY \& PIMENTEL (1985) observaram as mais altas fecundidades de $\boldsymbol{M}$. persicae (43,92 e 46,37 ninfas/ adulto, respectivamente), a $25^{\circ} \mathrm{C}$. Em estudo realizado por CIVIDANES \& SOUZA (2003) com $\boldsymbol{M}$. persicae sobre couve, foi observado que o aumento da temperatura prejudicou a fecundidade, com médias de 69,2; 63,3 e 30,7 ninfas/fêmea, respectivamente, nas temperaturas de 15,20 e $25^{\circ} \mathrm{C}$.

A temperatura influenciou de maneira inversa a fecundidade diária e a fecundidade total.
Enquanto o aumento da temperatura implicou o aumento da fecundidade diária, ocorreu um decréscimo da fecundidade total. Esta diferença pode ser explicada pela influência da temperatura na duração do período reprodutivo. A $15^{\circ} \mathrm{C}$, as fêmeas apresentaram maior período reprodutivo (19,11 dias), fazendo com que a produção de ninfas fosse distribuída ao longo de todo o período, diminuindo a média diária, mas resultando numa alta fecundidade total. A $25^{\circ} \mathrm{C}$, a duração do período reprodutivo foi bem menor (3,89 dias), fazendo com as fêmeas concentrassem sua reprodução, elevando a fecundidade diária, mas em função do curto período reprodutivo, a fecundidade diária foi menor (Tabela 2).

Período pós-reprodutivo.

A duração deste período decresceu com a elevação da temperatura, ajustando-se ao modelo quadrático (Figura 1). A maior duração desse período foi observada nas fêmeas mantidas a $15^{\circ} \mathrm{C}$, com 3,72 dias, diferindo das durações observadas nas temperaturas de 20 e $25^{\circ} \mathrm{C}$ (1,10 e 0,89 dia, respectivamente) (Tabela 2). Resultados semelhantes foram observados por XIA \& TINGEY (1986), quando analisados somente nas temperaturas de 20 e $25^{\circ} \mathrm{C}$, estudando $M$. persicae em dois híbridos de batata, "Superior” e D 888-4, observaram duração do período pós-reprodutivo de 2,83 dias e 0,96 dia nas temperaturas de $22: 15^{\circ} \mathrm{C}$ (dia/noite), respectivamente, e 1,00 e 0,63 dia nas temperaturas de 32:18 C (dia/noite) para os híbridos "Superior” e D 888-4, respectivamente. NARVÁEZ \& NOTZ (1993) observaram duração média do período pós-reprodutivo de 0,8 e 2,2 dias, para folhas de batata e gergelim, respectivamente, na temperatura de $26,71 \pm 3,02^{\circ} \mathrm{C}$.

Longevidade

A longevidade das fêmeas ápteras de $\boldsymbol{M}$. persicae em função da temperatura ajustou-se a um modelo linear (Figura 1), no qual a maior longevidade foi observada nos indivíduos mantidos a $15^{\circ} \mathrm{C}$, com 23,95 dias, e menor naqueles mantidos a $25^{\circ} \mathrm{C}$, com 5,06 dias (Tabela 1). CIVIDANES \& SOUZA (2003) observaram resultados superiores, com longevidade média de 26,7; 24,9 e 18,7 dias para as temperaturas de 15,20 e $25^{\circ} \mathrm{C}$, respectivamente.

Em Campinas, principal município produtor de berinjela (CEASA, 2004), a maior ocorrência de $\boldsymbol{M}$. persicae tem sido verificada durante os meses do ano em que as temperaturas estiveram entre 16 e $20^{\circ} \mathrm{C}$ (COSTA, 1970). Como neste trabalho as temperaturas de 15 e $20^{\circ} \mathrm{C}$ proporcionaram as maiores fecundidades totais, é necessário o monitoramento mais constante das plantas de berinjela nesta faixa de temperatura. 


\section{CONCLUSÕES}

O aumento da temperatura de 15 para $25^{\circ} \mathrm{C}$, proporciona a diminuição na duração dos períodos pré, pós e reprodutivo e na longevidade de fêmeas de $\boldsymbol{M}$. persicae sobre discos foliares de berinjela. $\mathrm{Na}$ temperatura de $15^{\circ} \mathrm{C}$, ocorre a maior fecundidade total. A temperatura de $30^{\circ} \mathrm{C}$ afeta negativamente a sobrevivência das fêmeas, impedindo-as de se reproduzirem.

\section{AGRADECIMENTOS}

Os autores agradecem à CAPES (Coordenadoria de Aperfeiçoamento de Pessoal de Nível Superior) pela bolsa concedida ao primeiro autor.

\section{REFERÊNCIAS}

BARLOW, C.A. The influence of temperature on the growth of experimental populations of Myzus persicae (Sulzer) and Macrosiphum euphorbiae (Thomas) (Aphididae). Canadian Journal of Zoology, Ottawa, v.40, n.1, p.145-156, 1962.

BASTOS, C.S. et al. Tabelas de fertilidade e esperança de vida de Myzus persicae (Sulzer) (Homoptera: Aphididae) em couve comum. Científica, São Paulo, v.24, n.1, p.187197, 1996.

BLACKMAN, R.L.; EASTOP, V.F. Aphids on the world's crops: an identification guide. Chichester : J. Wiley, 1984 466p.

CEASA. Padronização: berinjela. Capturado em 13 set. 2004. Online. Disponível na Internet http:// www.ceasacampinas.com.br/padronizacao_berinjela.htm.

CIVIDANES F.J., SOUZA V.P. Exigências térmicas e tabelas de vida de fertilidade de Myzus persicae (Sulzer) (Hemiptera: Aphididae) em laboratório. Neotropical Entomology, Londrina, v.32, n.3, p.413-419, 2003.

COSTA, C.L. Variações sazonais da migração de Myzus persicae em Campinas nos anos de 1967 a 1969. Bragantia, Campinas, v.29, n.1, p.347-360, 1970 .

CULLINEY, T.W.; PIMENTEL, D. The intrinsic rate of natural increase of the green peach aphid Myzus persicae (Sulzer) (Homoptera: Aphididae) on collards (Brassica oleracea L.). Canadian Entomologist, Ottawa, v.117, n.9, p.1147-1149, 1985.

DIXON, A.F.G. Parthenogenetic reproduction and the rate of increase in aphids. In : MINKS, A.K.; HARREWINJN, P. World crop pests-aphids: their biology, natural enemies and control. Amsterdam : Elsevier, 1987. V.2A, cap.4.5, p.97-287.

FILGUEIRA, F.A.R. Solanáceas III - Pimentão e outras hortaliças fruto. In: FILGUEIRA, F.A.R. Novo manual de olericultura. Viçosa : UFV, 2002. Cap.14, p.242-245.

GALLO, D. et al. Entomologia agrícola. Piracicaba : Fealq/ Esalq, 2002. V.10, 920p.

KOCOUREK, F. et al. Effect of temperature on development rate and intrinsic rate of increase of Aphis gossypii reared on greenhouse cucumbers. Entomologia Experimentalis et Applicata, Dordrecht, v.71, n.1, p.59-64, 1994.

MICHELOTTO, M.D.; BUSOLI, A. Aspectos biológicos de Aphis gossypii Glover, 1877 (Hemiptera: Aphididae) em três cultivares de algodoeiro e três espécies de plantas daninhas. Ciência Rural, Santa Maria, v.33, n.6, p.999-1004, 2003.

NARVÁEZ, Z.; NOTZ, A. Desarrollo, longevidad y reproduccion del afido verde del ajonjoli, Myzus persicae (Sulzer) (Homoptera: Aphididae) sobre plantas de papa (Solanum tuberosum L.) y ajonjoli (Sesamum indicum L.) Boletín de Entomología Venezolana, Maracay, v.8, n.1, p.53-61, 1993.

PINTO, C.M.F. et al. Doenças de berinjela e jiló. In: ZAMBOLIM, L.; et al. Controle de doenças de plantas e hortaliças. Viçosa : UFV, 2000. V.1, cap.10, p.303333.

SOGLIA, M.C.M. et al. Fecundidade e longevidade de Aphis gossypii Glover, 1877 (Hemiptera: Aphididae) em diferentes temperaturas e cultivares comerciais de crisântemo (Dendranthema grandiflora Tzvelev). Revista Brasileira de Entomologia, Curitiba, v.47, n.1, p.49-54, 2003.

VAN EMDEN, H.F. et al. The ecology of Myzus persicae. Annual Review of Entomology, Palo Alto, v.14, n.1, p.197239, 1969.

XIA, J., TINGEY., W.M. Green peach aphid (Homoptera: Aphididae): developmental and reproductive biology on Solanum tuberosum X S. berthaultii hybrid potato. Journal of Economic Entomololgy, New York, v.79, n.1, p.71-75, 1986. 